\title{
Vanillin production by biotransformation of phenolic compounds in fungus, Aspergillus luchuensis
}

\author{
Junsei Taira*, Rin Toyoshima, Nana Ameku, Akira Iguchi and Yasutomo Tamaki
}

\begin{abstract}
Vanillin is valuable and popular flavor used in foods and cosmetics. Many bacteria species have the ability to decarboxylate substituted cinnamic acids in order to form vanillin. However, the phenolic biotransformation including vanillin production in a common fungus, the Aspergillus luchuensis, which is used in distilled beverages, has not yet been clarified. This study focused on elucidating the vanillin production due to phenolic biotransformation in A. luchuensis during fermentation. The phenolic metabolites were extracted by a solid phase column and they were determined using on LC/MS and LC/MS/MS in a selective ion mode. As a result, ferulic acid, vanillin and vanillic acid, were detected in the rice koji fermentationed by $\mathrm{A}$. luchuensis and also fermentated with yeast. In addition, the accurate molecular formula of vanillin glucoside $\left(\mathrm{C}_{14} \mathrm{H}_{17} \mathrm{O}_{8}, 313.0927,(\mathrm{M}-\mathrm{H})^{-}\right.$and its production ions was also determined by HRESI-mass spectrometry. Based on the results including the phenolic metabolites and related genes found in A. luchuensis genome, this study proposed the vanillin production mechanism due to the side chain cleavage of ferulic acid through Coenzyme A (CoA) and feruloyl-CoA hydratase/lyase, to form vanillin and acetyl-COA. In this study, another possible vanillin production pathway also was proposed due to the neutral hexose hydrolysis of vanillin glucoside. The subsequent dehydrogenation of vanillin produced vanillic acid. In addition, vanillin was detected in the distilled alcohol indicating its contribution to the aroma profile of beverages. It has been unknown that the vanillin in the distilled solution is derived from the vanillin produced during rice-koji and/or moromi mash fermentations.
\end{abstract}

Keywords: Aspergillus species, Phenolic compound, Biotransformation, Vanillin, Ferulic acid, LC/MS

\section{Introduction}

Vanillin is an aromatic flavor compound which is frequently used in foods and cosmetics. Its production process by biotransformation has been intensively studied. Many bacteria species have the ability to decarboxylate substituted cinnamic acids, such as ferulic acid, eugenol, isoeugenol and $p$-coumaric acid, which are converted to useful aromatic chemicals, such as 4-vinyl guaiacol and 4-ethyl phenol (Priefert et al. 2001; Rabenhorst 1996; Narbad and Gasson 1998; Shimoni et al. 2000; Masai et al. 2002). Particularly, ferulic acid biocatalytically converted to produce vanillin, thus most attention has focused on the bioconversions of ferulic acid (Beek and

${ }^{*}$ Correspondence: taira@okinawa-ct.ac.jp Department of Bioresource Technology, Okinawa National College of Technology, 905 Henoko, Nago, Okinawa 905-2192, Japan
Priest 2000; Huang et al. 1993). Two types of ferulic acid side chain cleavages have been reported for a number of microorganisms (Rosazza et al. 1995). One type is catalyzed by nonoxidative decarboxylase, which eliminates one carbon from the ferulic acid side chain, resulting in the formation of 4-vinyl guaiacol. The other type is characterized by the elimination of two carbons from the ferulic acid side chain, and this latter reaction can produce vanillin, a valuable flavor compound. The role of lactic acid bacteria in the conversion of phenolic compounds to vanillin has been elucidated during the brewing of whisky and wine (Rosazza et al. 1995; Cavin et al. 1993; Bloem et al. 2007). East Asian countries, such as Japan, Philippine, China and Thailand have been using a common fungus for brewing. The Japanese spirit, shochu, is a traditional distilled beverage that uses a common fungus, the Aspergillus species, with the raw materials, such as rice, 
sweet potato or barley. Awamori is one type of shochu beverage that is produced from rice. During the production of awamori, rice-koji fermentation starter (tane-koji) of $A$. luchuensis is used in preparing the rice-koji, and following the completion of fermentation, the alcohol is distilled under ordinary pressure. Awamori shochu has a unique aroma profile, and recently, the characteristics of the natural vaporized aroma compounds emitted from awamori were elucidated (Taira et al. 2012). However, the presence of phenolic aroma compounds and the biotransformation have not yet been clarified. Therefore, this study focused on elucidating the biotransformation of phenolic compounds in A. luchuensis during fermentation. Consequently, the vanillin production mechanism in the Aspergillus species is for the first time proposed based on this study.

\section{Materials and methods}

\section{Chemicals}

Authentic compounds for analysis by mass spectrometry, such as hydroxy-3-methoxycinnamic acid (ferulic acid), 4-hydroxy-3-methoxybenzoic acid (vanillic acid), 4-hydroxy-3-methoxybenzaldehyde (vanillin) and 2-methoxy-4-vinylphenol (4-guaiachol) were commercially available. The rice-koji fermentation starter (tane koji) of A. luchuensis was obtained from Ishikawa Tanekoji Ten, Ltd. (Okinawa, Japan). Awamori 101 yeast was purchased from Shinzato-shuzo Co., Ltd. (Okinawa, Japan).

\section{Fermentation and alcohol distillation}

Japanese spirit, awamori shochu was produced by the general procedures as follows. Briefly, the washed long grain rice (Oryza sativa subsp., indica, $1200 \mathrm{~g}$ ) was immersed in water for 1 day. It was steamed by an autoclave treatment at $121{ }^{\circ} \mathrm{C}$ for $20 \mathrm{~min}$, and it was then cooled to $35^{\circ} \mathrm{C}$. The tane koji $(10 \mathrm{~g})$ was next added to the steamed rice and its rice-koji was prepared by incubation at $30{ }^{\circ} \mathrm{C}$ for $40 \mathrm{~h}$. To the rice-koji (400 g) was added mother water $(720 \mathrm{ml})$ and yeast (awamori 101 yeast, dry weight $0.1 \mathrm{~g}$ ), then the fermentation was carried out at $26^{\circ} \mathrm{C}$ for 15 days. Following the completion of fermentation, a $50 \%$ alcohol, except for the first drops for $5 \mathrm{~min}$, was distillated under decompression. Each analytical sample was collected from the rice-koji suspension, the fermentation products at 5 , 10, 15 days and the distilled alcohol. The samples of the rice-koji and fermentation product were centrifuged at $5000 \mathrm{rpm}$ for $30 \mathrm{~min}$ (HITACH himac CR22GII, Hitachi Koki, Co., Ltd., Tokyo, Japan). All samples were treated by a solid phase extraction step.

\section{Solid phase extraction}

The sample was extracted using a solid phase cartridge column (Oasis HLB Cartridge, Waters). Briefly, the column was conditioned with $5 \mathrm{ml}$ of methanol and MQ-water, then the sample $(5 \mathrm{ml})$ was loaded on the column. The column was continuously washed with $10 \mathrm{ml}$ of MQ-water and the sample was collected with $10 \mathrm{ml}$ of methanol. The solvent was evaporated at room temperature (Centrifugal evaporator CVE-310, Unitraput-1000, EYELA) and the residue dissolved in ethanol $(1 \mathrm{ml})$ was used as the analytical sample.

\section{LC/MS analysis}

Each sample was measured by LC/MS (Agilent1200, Agilent Technologies) using a photodiode array detector and monitored at $280 \mathrm{~nm}$ on a reversed-phase chromatographic column, YMC-Pack Pro C18 $(100 \times 4.6 \mathrm{~mm}$ I.D., $5 \mu \mathrm{m}$ particle size, YMC Co., Ltd., Japan) at $40.0{ }^{\circ} \mathrm{C}$. The mobile phase consisting of a $5 \mathrm{mM}$ formic acid aqueous solution $(10 \%)$ and acetonitrile was carried out at the flow rate of $0.8 \mathrm{ml} / \mathrm{min}$ by a linear gradient to $50 \%$ (10 min) and 100\% (5 min) and held for $5 \mathrm{~min}$. The mass spectra were measured under the following conditions: ESI negative ion mode; desolvation temperature, $350{ }^{\circ} \mathrm{C}$; desolvation pressure, 35 psig and desolvation gas flow, $12.01 \mathrm{ml} / \mathrm{min}$ (6120 Quadrupole, Agilent Technologies).

\section{LC/MS/MS analysis}

The HRESI-mass spectra were measured using an LC/ MS/MS (Agilent 6560 IM-QTOF, Agilent Technologies). The LC was then carried out under the same conditions for the LC/MS analysis. The mass spectra were measured under the following conditions: ESI negative ion mode; drying gas temperature, $350{ }^{\circ} \mathrm{C}$; desolvation pressure, $35 \mathrm{psig}$ and desolvation gas flow, $12 \mathrm{ml} / \mathrm{min}$; capillary voltage, $3500 \mathrm{~V}$; nozzle voltage, $500 \mathrm{~V}$; collision, $20 \mathrm{~V}$. Reference ions were used for 19.03632 and 966.000725 .

\section{Bioinformatics}

The annotation information of amino acid sequences from A. luchuensis genome (accession nos: BCWF01000001-BCWF01000044, Yamada et al. 2016) was searched using BLASTP against the Swiss-Prot and TrEMBL protein databases from Uniprot (e-value cutoff 1e-5). Candidate genes related to vanillin production were searched based on the keywords, such as vanillin, enoyl (feruloyl), glucosidase and glucosyl transferase.

\section{Results}

\section{Identification of phenolic compounds}

In this study investigated the phenolic biotransformation including vanillin production due to phenolic biotransformation in A. luchuensis during fermentation together 
with brewing. The phenolic compounds in A. luchuensis during fermentation were detected by LC/MS and LC/ MS/MS (Fig. 1). Each total ion chromatogram (TIC) of ferulic acid, vanillin, vanillic acid and vanillin glucoside is shown in Fig. 2. The molecular weights and retention time of ferulic acid $\left(193.2,(\mathrm{M}-\mathrm{H})^{-}, 6.95 \mathrm{~min}\right)$, vanillin $\left(151.2,(\mathrm{M}-\mathrm{H})^{-}, 4.94 \mathrm{~min}\right)$ and vanillic acid (167.1, $\left.(\mathrm{M}-\mathrm{H})^{-}, 5.89 \mathrm{~min}\right)$ were identical to those of the authentic compounds except for vanillin glucodide. A similar molecular weight $\left((\mathrm{M}-\mathrm{H})^{-}, 313.3,4.33 \mathrm{~min}\right)$ for vanillin glucoside was also obtained from the mash and its fermentation product. The HRESI-mass spectrum of vanillin glucoside was measured to clarify the molecule by LC/MS/MS (Fig. 3). The accurate molecular formula of vanillin glucoside was determined to be $\mathrm{C}_{14} \mathrm{H}_{18} \mathrm{O}_{8}$ based on the mass of $313.0927(\mathrm{M}-\mathrm{H})^{-}, 313.0926$, calcd. for $\mathrm{C}_{14} \mathrm{H}_{18} \mathrm{O}_{8}$. The other production ions derived from the vanillin $(\mathrm{m} / z$ 161.0588, 177.0557, 180.0426 and 205.0495) were also detected and they were structurally supported to be vanillin glucoside.

\section{Changes of the phenolic compounds during fermentation} The contents of these phenolic compounds in the rice mold (rice-koji) without yeast, its fermentation products for 5, 10 and 15 days and the distilled alcohol are indicated in Fig. 4. The phenolic compounds, such as ferulic acid, vanillin and vanillic acid, were detected in the ricekoji and during fermentation, and vanillin and vanillic

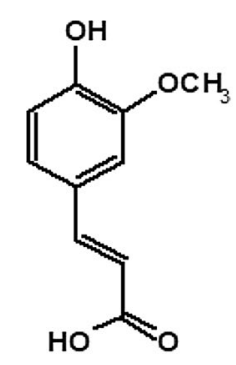

Ferulic acid

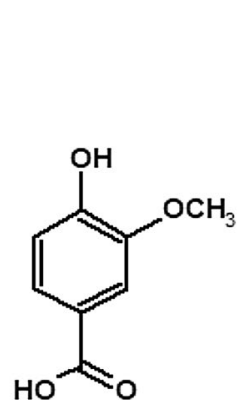

Vanillic acid

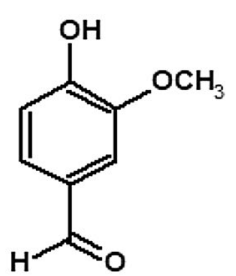

Vanillin

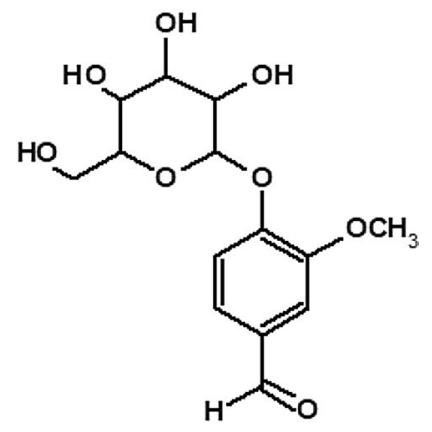

Vanillin glucoside

Fig. 1 The phenolic compounds were detected in rice-koji fermented by Aspergillus luchuensis acid also transferred to the distilled alcohol. These phenolic compounds were produced in the rice-koji and during the fermentation, indicating the metabolites due to phenolic biotransformation in A. luchuensis. A previous study reported the detection of phenolic compounds, such as 4-vinyl alcohol, vanillin and vanillic acid are only detected in the distilled awamori shochu or its aging (Koseki et al. 1996). Therefore, the phenolic compounds during the biotransformation of A. luchuensis were first determined in this study.

Esterified ferulic acid in the cell walls of crops is hydrolyzed by feruloyl esterase. The esterases that the hydrolyzed the ester linkages of ferulic acid in the cell walls have been isolated from Streptomyces olivochromogenes (Craig and Williamson 1991), A. niger (Craig et al. 1997) and $A$. luchuensis (Koseki et al. 1998). In this study, the ferulic acid was detected in the steam riced materials before the mash, indicating that the ferulic acid is already present in the raw materials (data not shown), then it was accumulated by feruloyl esterase during the fermentation (Fig. 4).

\section{Bioinformatics for phenolic compounds}

The annotation information of amino acid sequences from $A$. luchuensis genome searched using BLAST. Particularly, the related genes to the vanillin production was investigated, and then the possible 82 genes contribute to the phenolic biotransformation were found in the genome information of $A$. luchuensis (Additional file 1: Table S1). Specifically, the genes of feruloyl estelases, enoyl (feruloyl)-CoA synthases, enoyl (feruloyl)-CoA hydratases suggested that they are take part in the vanillin production, and also vanillin dehydrogenase contributes to vanillic acid production due to dehydrogenation of vanillin. In addition, the genes related to glucosidase and glucose transferase suggested another possible pathway to be produced vanillin from vanillin glucoside which was detected in the biotransformation of $A$. luchuensis during fermentation (Fig. 4).

\section{Discussion}

It has been reported that the decarboxylation of ferulic acid due to many lactic acid bacteria strains produces 4-vinyl guaiachol coupled with 4-ethyl phenol and the following oxidation of 4-vinyl guaiachol produced vanillin during the fermentation (Beek and Priest 2000; Huang et al. 1993; Rosazza et al. 1995). In this study, these decarboxylated compounds due to the one carbon elimination of the ferulic acid side chain were confirmed, but it was not detected in any sample. Thus, the vanillin production pathway with decarboxylation will not be suitable for the biotransformation in A. luchuensis. Previous articles reported that the elimination of two carbons from 

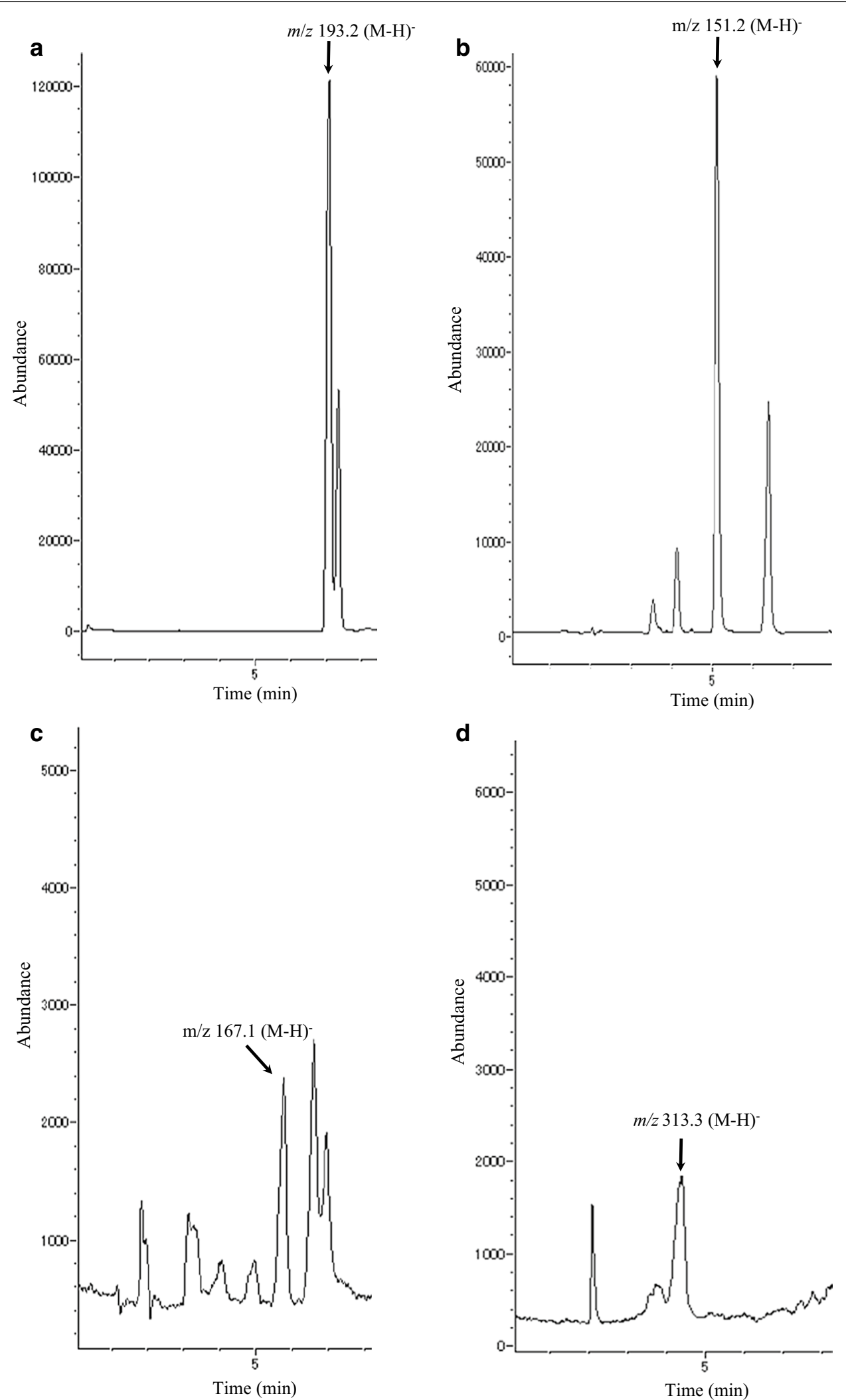

Fig. 2 Total ion chromatogram obtained from the biotransformation of phenolic compounds in Aspergillus /uchuensis. The total ion chromatogram (TIC) was measured by LC/MS. Each TIC mass spectrum for a ferulic acid $\left((\mathrm{M}-\mathrm{H})^{-}, 193.2\right)$, b vanillin $\left((\mathrm{M}-\mathrm{H})^{-}{ }^{-}, 151.2\right)$, c vanillic acid $\left((\mathrm{M}-\mathrm{H})^{-},{ }^{-}, 167.1\right), \mathbf{d}$ vanillin glucoside $\left((\mathrm{M}-\mathrm{H})^{-}, 313.3\right)$ and individual retention times were identical to those of the authentic compounds 


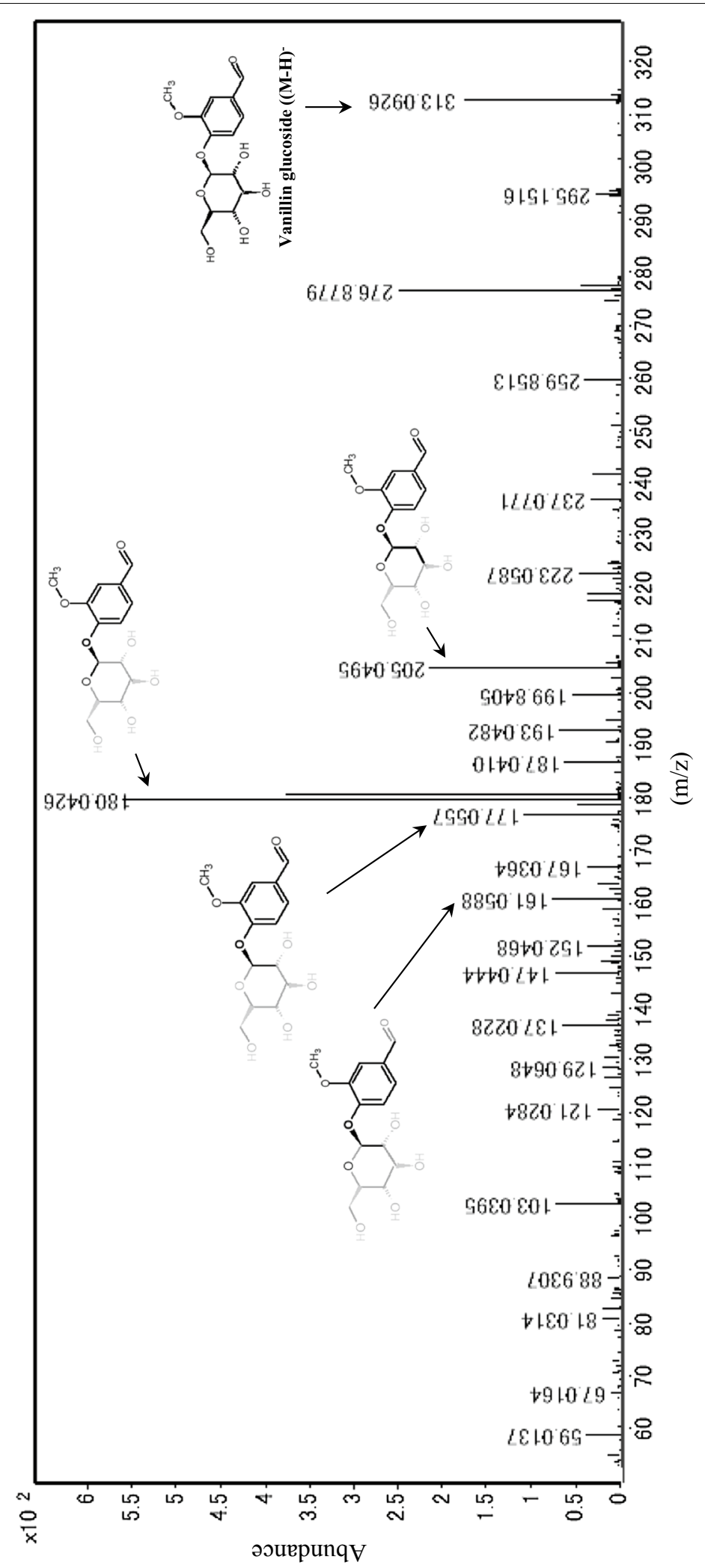

Fig. 3 The mass spectrometry of vanillin glucoside produced during the phenolic biotransformation of Aspergillus luchuensis. The HRESI-mass spectrometry of vanillin glucoside was measured by LC/MS/MS. The accurate molecular formula of vanillin glucoside was determined to be $\mathrm{C}_{14} \mathrm{H}_{18} \mathrm{O}_{8}$ based on the mass $313.0927(\mathrm{M}-\mathrm{H})^{-}, 313.0926$, calcd. for $\mathrm{C}_{14} \mathrm{H}_{18} \mathrm{O}_{8}$. The other production ions derived from the vanillin $(\mathrm{m} / \mathrm{z}: 161.0588,177.0557$, 180.0426 and 205.0495) were also detected which were structurally supported 


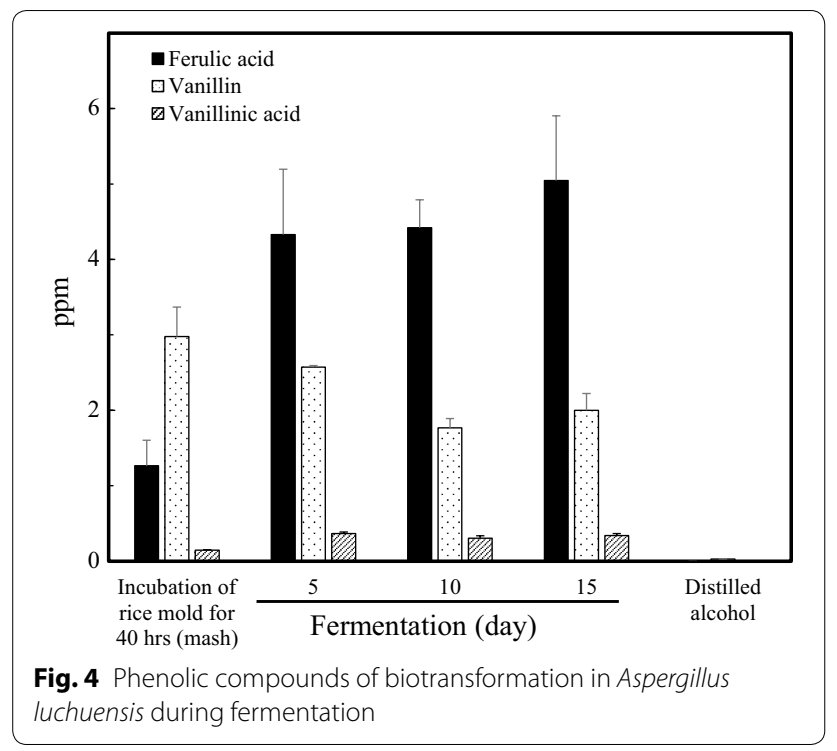

the ferulic acid side chain through the hydration and nonoxidative cleavage of feruloyl-CoA (enoyl-CoA) produced vanillin (Narbad and Gasson 1998; Gasson et al. 1998; Overhage et al. 1999). Briefly, the feruloyl-CoA synthetase catalyzes the transfer of CoA to the carboxyl group of ferulic acid, which then forms feruloyl-CoA. The feruloyl-CoA is degraded by the feruloyl-CoA hydratases (enoyl-CoA hydratases)/lyases to form 4-hydroxy3-methoxyphenyl-hydroxypropionyl-CoA, and its cleavage produces vanillin and acetyl-COA. The produced vanillin from the biotransformation will be dehydrogenated to vanillic acid by vanillin dehydrogenase (Rosazza et al. 1995). The related genes to the vanillin production, such as feruloyl esterase, enoyl-CoA hydratases, vanillin dehydrogenase, glucosidases and glucosyltransferases were detected in the genome information of A. luchuensis (Additional file 1: Table S1). The expression genes and related to the enzymes which contribute to the vanillin production pathway will be clarified in our future study.

Based on the results including phenolic metabolites and the related genes, this study proposes a similar phenolic biotransformation mechanism that involves in a two-step process, the CoA ligases followed by the sidechain cleavage of ferulic acid through feruloyl-CoA and feruloyl-CoA hydratase/lyase give vanillin and acetyl$\mathrm{COA}$, then the dehydrogenation of vanillin produced vanillic acid (Fig. 5).

Interestingly, the vanillin glucoside was also first identified in the Aspergillus species in the mash and its fermentation. As shown in the vanillin production scheme (Fig. 5), glucose hydrolysis of vanillin glucoside would be produced vanillin through the reversible reactions in the presence of glucosidase and glucosyltransferase (Gallage et al. 2014). This pathway may take part in one of the vanillin production mechanisms. In addition, the vanillin during fermentation was also detected in the distilled alcohol indicating its contribution to the aroma profile of the awamori shochu.

In conclusion, this study proposed the phenolic biotransformation mechanism in $A$. luchuensis that is involves a two-step process, i.e., the CoA ligases followed by a two-carbon elimination of ferulic acid produce vanillin and acetyl-COA. Also, another vanillin production pathway derived from the vanillin glucoside was proposed. The following dehydrogenation of vanillin will produce vanillic acid. In addition, vanillin in the distilled alcohol indicated its contribution to the aroma of shochu. 


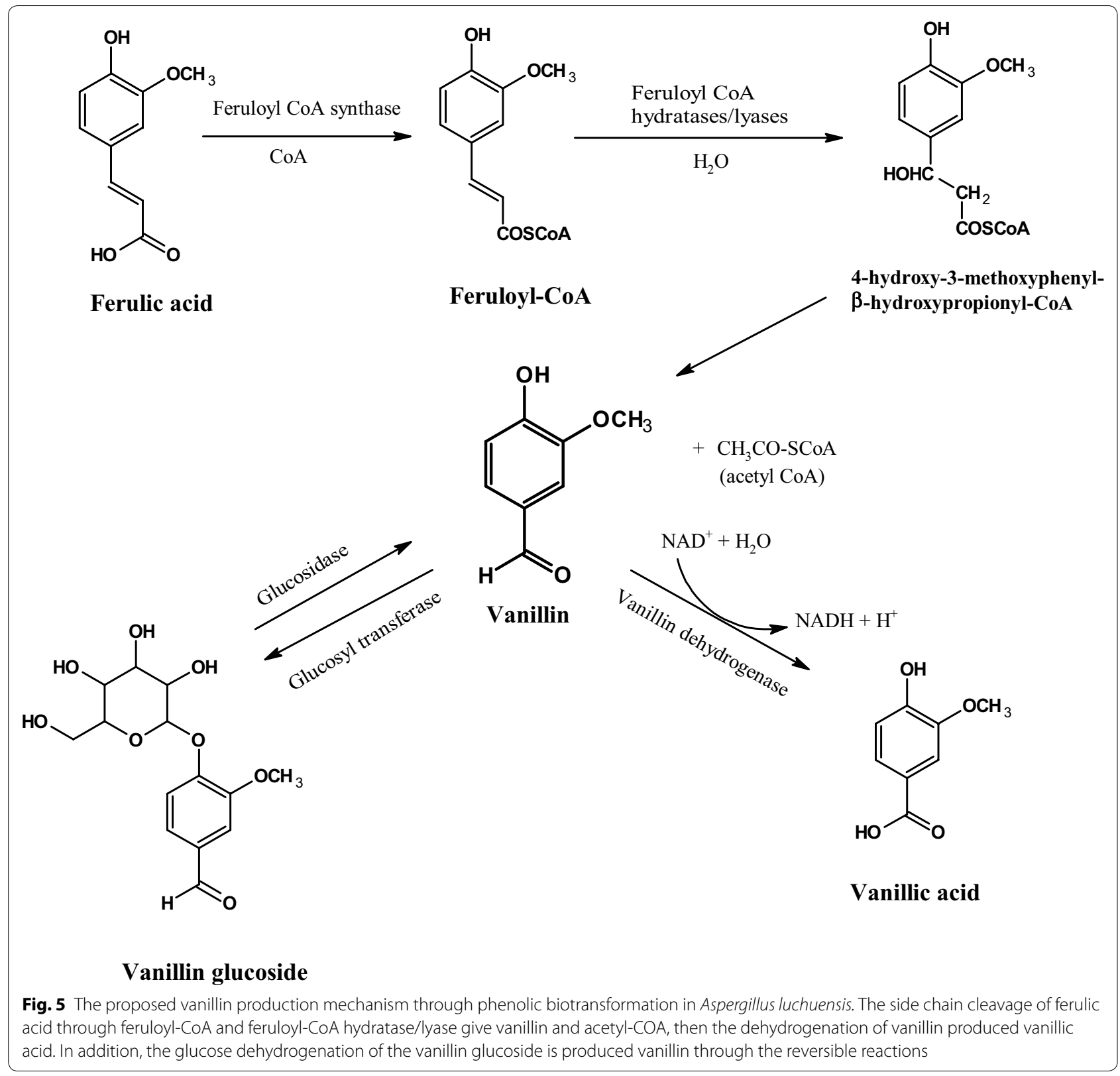

\section{Additional file}

Additional file 1: Table S1. Candidate genes for vanillin production found in Aspergillus luchuensis genome.

\section{Abbreviations}

LC/MS: liquid chromatography-mass spectrometry; ESI: electrospray ionization; LC/MS/MS: liquid chromatography-tandem mass spectrometry; HRESI: high-resolution-electrospray ionization.
Authors' contributions

JT conceived and designed the study. RT, NA, YT, JT performed the experiments and Al studied the Bioinformatics. JT wrote the paper and Al reviewed.

JT edited the manuscript. All authors read and approved the final manuscript.

\section{Acknowledgements}

We thank Drs. Konno, Y and Hayashi A for measuring HRESI-mass spectrometry (Agilent Technologies).

\section{Competing interests}

The authors declare that they have no competing interests. 


\section{Availability of data and materials}

All datasets supporting the conclusions of the manuscript were included in the manuscript.

\section{Ethics approval and consent to participate}

This article does not contain any studies with human participants or animals performed by any of the authors.

\section{Funding}

Not applicable for that section.

\section{Publisher's Note}

Springer Nature remains neutral with regard to jurisdictional claims in published maps and institutional affiliations.

Received: 27 December 2017 Accepted: 8 March 2018

Published online: 13 March 2018

\section{References}

Beek SV, Priest FG (2000) Decarboxylation of substituted cinnamic acids by lactic acid bacteria isolated during malt whisky fermentation. Appl Environ Microbiol 66:5322-5328

Bloem A, Bertrand A, Lonvaud-Funel A, de Revel G (2007) Vanillin production from simple phenols by wine-associated lactic acid bacteria. Lett Appl Microbiol 44:62-67

Cavin JF, Andioc V, Etievant PX, Divies C (1993) Ability of wine lactic acid bacteria to metabolize phenol carboxylic acids. Am J Enol Vitic 44:76-80

Craig BF, Williamson G (1991) The purification and characterization of 4-hydroxy-3-methoxycinnamic (ferulic) acid esterase from Streptomyces Olitrochromogenes. J Gen Microbiol 137:2339-2345

Craig BF, deVries P, Ronald AK, Paul Jaap V (1997) Influence of ferulic acid on the production of feruoyl estelase by Aspergillus niger. FEMS Microbiol Lett 157:239-244

Gallage NJ, Hansen EH, Kannangara R, Olsen CE, Motawia MS, Jorgensen K, Holme I, Hebelstrup K, Grisoni M, Moller BL (2014) Vanillin formation from ferulic acid in Vanilla planifolia is catalysed by a single enzyme. Nat Commun. https://doi.org/10.1038/ncomms5037

Gasson MJ, Kitamura Y, McLauchlan WR, Narbad A, Parr AJ, Parsons ELH, Payne J, Rhodes MJC, Waltoni NJ (1998) Metabolism of ferulic acid to vanillin
A bacterial gene of the enoyl-SCoA hydratase superfamily encodes an enzyme for the hydration and cleavage of a hydroxycynamic acid SCOA thioester. J Biol Chem 273:4163-4170

Huang Z, Dostal L, Rosazza JPN (1993) Microbial transformations of ferulic acid by Saccharomyces cerevisiae and Pseudomonas fluorescens. Appl Environ Microbiol 59:2244-2250

Koseki T, Ito Y, Furuse S, Ito K, Iwano K (1996) Conversion of ferulic acid into 4-vinylaclcohol, vanillin and vanillic acid in model solutions of shochu awamori. J Ferment Bioeng 82:46-50

Koseki T, Furuse S, Iwano K, Matsuzawa H (1998) Purificaton and characterization of a feruoylessterase in Aspergillus awamori. Biosci Biotechnol Biochem 62:2032-2034

Masai E, Harada K, Peng X, Kitayama H, Katayama Y, Fukuda M (2002) Cloning and characterization of the ferulic acid catabolic genes of Sphingomonas paucimobilis SYK-6. Appl Environ Microbiol 68:4416-4424

Narbad A, Gasson MJ (1998) Metabolism of ferulic acid via vanillin using a novel CoA-dependent pathway in a newly-isolated strain of Pseudomonas fluorescens. Microbiology 144:1397-1405

Overhage J, Priefert H, Steinbüchel A (1999) Biochemical and genetic analyses of ferulic acid catabolism in Pseudomonas sp. strain HR199. Appl Environ Microbiol 65:4837-4847

Priefert H, Rabenhorst J, Steinbüchel A (2001) Biotechnological production of vanillin. App Microbiol Biotechnol 56:296-314

Rabenhorst J (1996) Production of methoxyphenol-type natural aroma chemicals by biotransformation of eugenol with a new Pseudomonas sp. Appl Microbiol Biotechnol 46:470-474

Rosazza JP, Huang Z, Dostal L, Volm T, Rousseau B (1995) Review: biocatalytic transformations of ferulic acid: an abundant aromatic natural product. J Ind Microbiol 15:457-471

Shimoni E, Ravid U, Shoham Y (2000) Isolation of a Bacillus sp. capable of transforming isoeugenol to vanillin. J Biotechnol 78:1-9

Taira J, Tsuchiya A, Furudate H (2012) Initial volatile aroma profiles of young and aged awamori Shochu determined by GC/MS/Pulsed FPD. Food Sc Technol Res 18:177-181

Yamada O, Machida M, Hosoyama A, Goto M, Takahashi T, Futagami T, Yamagata Y, Takeuchi M, Kobayashi T, Koike H, Abe K, Asai K, Arita M, Fujita N, Fukuda K, Higa K, Horikawa H, Ishikawa T, Jinno K, Kato Y, Kirimura K, Mizutani O, Nakasone K, Sano M, Shiraishi Y, Tsukahara M, Gomi K (2016) Genome sequence of Aspergillus luchuensis NBRC 4314. DNA Res 23:507-515

\section{Submit your manuscript to a SpringerOpen ${ }^{\circledR}$ journal and benefit from:}

- Convenient online submission

- Rigorous peer review

- Open access: articles freely available online

- High visibility within the field

- Retaining the copyright to your article

Submit your next manuscript at springeropen.com 